\title{
Solving parabolic integro-differential equations with purely nonlocal conditions by using the operational matrices of Bernstein polynomials
}

\author{
Abdelkrim Bencheikh ${ }^{\mathrm{a}, *}$, Lakhdar Chiter ${ }^{\mathrm{b}}$, Tongxing Li $\mathrm{Li}^{\mathrm{c}, \mathrm{d}}$ \\ ${ }^{a}$ Department of Mathematics, University of Ouargla, 30000 Ouargla, Algeria. \\ ${ }^{b}$ Department of Mathematics, University of Setif 1, 19000 Setif, Algeria. \\ ${ }^{c}$ LinDa Institute of Shandong Provincial Key Laboratory of Network Based Intelligent Computing, Linyi University, Linyi, Shandong \\ 276005, P. R. China. \\ ${ }^{d}$ School of Information Science and Engineering, Linyi University, Linyi, Shandong 276005, P. R. China.
}

Communicated by K. Q. Lan

\begin{abstract}
Some problems from modern physics and science can be described in terms of partial differential equations with nonlocal conditions. In this paper, a numerical method which employs the orthonormal Bernstein polynomials basis is implemented to give the approximate solution of integro-differential parabolic equation with purely nonlocal integral conditions. The properties of orthonormal Bernstein polynomials, and the operational matrices for integration, differentiation and the product are introduced and are utilized to reduce the solution of the given integro-differential parabolic equation to the solution of algebraic equations. An illustrative example is given to demonstrate the validity and applicability of the new technique.
\end{abstract}

Keywords: Integro-differential parabolic equation, purely nonlocal integral conditions, orthonormal Bernstein polynomials, operational matrix.

2010 MSC: 33C45, 35K20, 42C05.

(C)2018 All rights reserved.

\section{Introduction}

In this paper, we are concerned with a class of parabolic integro-differential equations

$$
v_{t}(x, t)-v_{x x}(x, t)=\int_{0}^{t} a(t-\tau) v(x, \tau) d \tau, \quad 0<x<1, \quad 0<t \leqslant T
$$

subject to the initial condition

$$
v(x, 0)=\Phi(x), \quad 0<x<1
$$

\footnotetext{
*Corresponding author

Email addresses: krimbench@yahoo.fr (Abdelkrim Bencheikh), 1chiter@univ-setif .dz (Lakhdar Chiter),

litongx2007@163.com (Tongxing Li)
}

doi: $10.22436 /$ jnsa.011.05.04

Received: 2017-03-21 Revised: 2017-11-18 Accepted: 2018-03-01 
and the purely nonlocal (integral) conditions

$$
\int_{0}^{1} v(x, t) d x=r(t), \quad \int_{0}^{1} x v(x, t) d x=q(t), \quad 0<t \leqslant T,
$$

where $v$ is an unknown function, $r, q$, and $\Phi$ are given functions supposed to be sufficiently regular, a is suitably defined function satisfying certain conditions that will be specified later, and $\mathrm{T}$ is a positive constant.

Boundary value problems with integral boundary conditions are an interesting and important class of problems; see, e.g., [2, 4, 5, 8-10, 13, 14, 22]. For instance, the nonlocal term of our problem, i.e., $\int_{0}^{t} a(t-\tau) v(x, \tau) d \tau$, appears in the modeling of the quasi-static flexure of a thermo-elastic rod [12]. First, this problem with the more general second-order parabolic equation or a $2 \mathrm{~m}$-th order parabolic equation has been studied using the energy-integral methods and Rothe method in [11, 12] and [17], respectively. For other models, we refer the reader to $[6,18,21]$. The problem (1.1)-(1.3) is studied by using the Rothe method in [13], the existence and uniqueness of solution to this problem is given in [15], where the proofs are based on a priori estimates and Laplace transform method. On the other hand, in [1], the author considered a one-dimensional heat equation with nonlocal integral conditions and applied the Laplace transform to the problem. Similar problem can be found in [14]. Recently, various types of partial differential equations (PDEs) with nonlocal conditions have been studied in [2-4, 10, 11, 18, 19] among others, and the use of nonlocal conditions was extended to cover a wide variety of PDEs, and integro-differential equations; see, e.g., [13, 15, 16, 22].

This paper is organized as follows. In the next section, we describe the basic formulation of orthonormal Bernstein polynomials (OBPs) and their expansion in terms of Taylor basis, and we explain the general procedure of operational matrices of integration, differentiation and product, respectively. In Section 3, we transform the problem (1.1)-(1.3) to an equivalent one with homogeneous integral conditions, namely, problem (3.1)-(3.2), and we solve it by the use of operational matrix of OBPs. An illustrative numerical example is given in sub-section 3.2 to demonstrate the viability and practical usefulness of the method. The paper ends in Section 4 with some concluding remarks.

\section{Properties of orthonormal Bernstein polynomials}

The explicit representation of the orthonormal Bernstein polynomials of $m^{\text {th }}$ degree are defined on the interval $[0,1]$ in [7] by

$$
\mathrm{OB}_{j, m}(x)=\sqrt{2(m-j)+1}(1-x)^{m-j} \sum_{k=0}^{j}(-1)^{k}\left(\begin{array}{c}
2 m+1-k \\
j-k
\end{array}\right)\left(\begin{array}{l}
j \\
k
\end{array}\right) x^{j-k}, j=0, \ldots, m .
$$

In addition, (2.1) can be written in a simpler form in terms of original non-orthonormal Bernstein basis functions as follows:

$$
\mathrm{OB}_{j, m}(x)=\sqrt{2(m-j)+1} \sum_{k=0}^{j}(-1)^{k} \frac{\left(\begin{array}{c}
2 m+1-k \\
j-k
\end{array}\right)\left(\begin{array}{l}
j \\
k
\end{array}\right)}{\left(\begin{array}{c}
m-k \\
j-k
\end{array}\right)} B_{j-k, m-k}(x), \quad j=0, \ldots, m .
$$

These polynomials satisfy the following orthogonality relation

$$
\int_{0}^{x} \mathrm{OB}_{i, m}(x) \mathrm{OB}_{j, m}(x) d t=\delta_{i j}, \quad i, j=0, \ldots, m,
$$

where $\delta_{i j}$ is the Kronecker delta function. 
2.1. Expansion of OBPs in terms of Taylor basis

Using (2.1) and (2.2), we have, for $x \in[0,1]$ and $i=0, \ldots, m$,

$$
\begin{aligned}
\mathrm{OB}_{j, m}(x) & =\sqrt{2(m-j)+1}\left(\sum_{r=0}^{m-i} \alpha_{i, r} x^{r}\right)\left(\sum_{j=0}^{i} \beta_{i, j} x^{j}\right) \\
& =\sqrt{2(m-j)+1} \sum_{j=0}^{m}\left(\sum_{k=\max \{0, j-m+i\}}^{\min \{i, j\}} \alpha_{i, j-k} \beta_{i, k}\right) x^{j},
\end{aligned}
$$

where

$$
\begin{aligned}
& \alpha_{i, r}=(-1)^{r}\left(\begin{array}{c}
m-i \\
r
\end{array}\right), \quad r=0, \ldots, m-i, \\
& \beta_{i, j}=(-1)^{i-j}\left(\begin{array}{c}
2 m+1-i+j \\
j
\end{array}\right)\left(\begin{array}{c}
i \\
i-j
\end{array}\right), \quad j=0, \ldots, i .
\end{aligned}
$$

Equation (2.3) can be displayed in the following matrix form

$$
\mathrm{OB}(x)=\mathrm{MT}_{m}(x), \quad x \in[0,1],
$$

where

$$
M_{i, j}=\sqrt{2(m-j)+1} \sum_{k=\max 0, j-m+i}^{\min i, j} \alpha_{i, j-k} \beta_{i, k}, i, j=0, \ldots, m
$$

and

$$
\mathrm{OB}(x)=\left[\mathrm{OB}_{0, \mathrm{~m}}(x), \mathrm{OB}_{1, \mathrm{~m}}(x), \ldots, \mathrm{OB}_{\mathrm{m}, \mathrm{m}}(x)\right]^{\top}, \quad \mathrm{T}_{\mathrm{m}}(\mathrm{x})=\left[1, x, \ldots, x^{\mathrm{m}}\right]^{\top} .
$$

\subsection{Function approximation}

Given an interval $I=(a, b)$ and a generic positive weight function $\omega$ on $I$. Let $P_{m}, m \geqslant 1$ be the space of polynomials degree $\leqslant m$, and the sequence $\left\{\varphi_{j}\right\}_{j=0}^{\infty}$ be a complete orthonormal set of functions in $\mathrm{L}_{\boldsymbol{\omega}}^{2}(\mathrm{I})$ with $\varphi_{j} \in \mathrm{P}_{j}, j=0,1, \ldots$ The inner product and its corresponding norm in $\mathrm{L}_{\omega}^{2}$ (I) are defined by

$$
\langle u, v\rangle_{\omega}=\int_{a}^{b} u(t) v(t) \omega(t) d t \quad \text { and }\|u\|_{L_{\omega}^{2}}=\sqrt{\langle u, v\rangle_{\omega}} \text { for all } u, v \in L_{\omega}^{2}(I) .
$$

Theorem 2.1 ([20]). For any $\mathrm{u} \in \mathrm{L}_{\omega}^{2}$ (I) and $\mathrm{m} \geqslant 1$, there exists a unique $\mathrm{q}_{\mathrm{m}}^{*} \in \mathrm{P}_{\mathrm{m}}$ such that

$$
\left\|\mathrm{u}-\mathrm{q}_{\mathrm{m}}^{*}\right\|_{\mathrm{L}_{\omega}^{2}}=\inf _{\mathrm{q}_{\mathrm{m}} \in \mathrm{P}_{\mathrm{m}}}\left\|\mathrm{u}-\mathrm{q}_{\mathrm{m}}^{*}\right\|_{\mathrm{L}_{\omega}^{2}} \text {, }
$$

where

$$
\mathrm{q}_{\mathrm{m}}^{*}(x)=\sum_{\mathrm{k}=0}^{\mathrm{m}} \hat{\mathrm{u}}_{\mathrm{k}} \mathrm{p}_{\mathrm{k}}(x)
$$

with

$$
\hat{u}_{k}=\frac{\left\langle u, p_{k}\right\rangle_{\omega}}{\left\|p_{k}^{2}\right\|}
$$

and $\left\{\mathrm{p}_{\mathrm{k}}\right\}_{\mathrm{k}=0}^{\mathrm{m}}$ forms an $\mathrm{L}_{\omega}^{2}$-orthogonal basis for $\mathrm{P}_{\mathrm{m}}$.

In particular, we denote the best approximation polynomial $\mathrm{q}_{\mathrm{m}}^{*}$ by $\pi_{\mathrm{m}} \mathrm{u}$, which is the $\mathrm{L}_{\omega}^{2}$-orthogonal projection of $u$. The OBPs are orthogonal with respect to the weight function $\omega(x)=1$ over $I=(0, L)$. Therefore, if $f$ is an arbitrary element in $\mathrm{L}_{2}(0, \mathrm{~L})$, then by Theorem $2.1, f$ has the unique best approximation $\pi_{\mathrm{m}} \mathrm{f}$ such that

$$
\pi_{\mathrm{m}} f=\sum_{\mathrm{k}=0}^{\mathrm{m}} \mathrm{c}_{\mathrm{k}} \varphi_{\mathrm{k}, \mathrm{m}}
$$

By virtue of (2.5) and (2.6), we get

$$
c_{k}=\left\langle f, \varphi_{k, m}\right\rangle, \quad k=0, \ldots, m .
$$


2.3. OBPs operational matrix of integration

Let $P$ be an $(m+1) \times(m+1)$ operational matrix of integration. Then

$$
\int_{0}^{x} \mathrm{OB}(\mathrm{t}) \mathrm{dt} \simeq \mathrm{P} \cdot \mathrm{OB}(\mathrm{x}), \quad 0 \leqslant x \leqslant 1 .
$$

By (2.4), we have

$$
\int_{0}^{x} \mathrm{OB}(\mathrm{t}) \mathrm{dt}=\mathrm{M}\left[\begin{array}{ccccc}
1 & 0 & 0 & \cdots & 0 \\
0 & \frac{1}{2} & 0 & \cdots & 0 \\
\vdots & \vdots & \vdots & \ddots & \vdots \\
0 & 0 & 0 & \cdots & \frac{1}{m+1}
\end{array}\right]\left[\begin{array}{c}
x \\
x^{2} \\
\vdots \\
x^{m+1}
\end{array}\right]=M \wedge X
$$

where $\Lambda$ is $(m+1) \times(m+1)$ matrix

$$
\Lambda=\left[\begin{array}{ccccc}
1 & 0 & 0 & \cdots & 0 \\
0 & \frac{1}{2} & 0 & \cdots & 0 \\
\vdots & \vdots & \vdots & \ddots & \vdots \\
0 & 0 & 0 & \cdots & \frac{1}{m+1}
\end{array}\right]
$$

and $X=\left[\begin{array}{llll}x & x^{2} & \ldots & x^{m+1}\end{array}\right]^{\top}$. Now, we approximate the elements of vector $X$ in terms of $\left\{\mathrm{OB}_{j, m}\right\}_{j=0}^{m}$. It follows from (2.4) that $T_{m}(x)=M^{-1} O B(x)$, and so for $k=0,1, \ldots, m$,

$$
x^{k}=M_{k+1}^{-1} \mathrm{OB}(x),
$$

where $M_{k+1}^{-1}$ is $(k+1)^{\text {th }}$ row of $M^{-1}$ for $k=0,1, \ldots, m$, that is,

$$
M^{-1}=\left[\begin{array}{c}
M_{1}^{-1} \\
M_{2}^{-1} \\
\vdots \\
M_{k+1}^{-1}
\end{array}\right]
$$

Hence, we only need to approximate $x^{m+1}$. Using (2.7), we obtain $x^{m+1}=c_{m+1}^{T} O B(x)$, where

$$
c_{m+1}=\int_{0}^{1} x^{m+1} \mathrm{OB}(\mathrm{t}) \mathrm{dt}
$$

Then

$$
X=\left[\begin{array}{c}
M_{2}^{-1} \\
M_{3}^{-1} \\
\vdots \\
M_{m+1}^{-1} \\
c_{m+1}^{T}
\end{array}\right] \text { OB }(x)
$$

Let

$$
B=\left[\begin{array}{c}
M_{2}^{-1} \\
M_{3}^{-1} \\
\vdots \\
M_{m+1}^{-1} \\
c_{m+1}^{\top}
\end{array}\right],
$$

we have

$$
\int_{0}^{x} \mathrm{OB}(\mathrm{t}) \mathrm{dt}=\mathrm{M} \wedge \mathrm{B} \cdot \mathrm{OB}(\mathrm{x}) \text {. }
$$

Therefore, we get the operational matrix of integration as $\mathrm{P}=\mathrm{M} \wedge \mathrm{B}$. 


\subsection{OBPs operational matrix of derivative}

In this section, we will derive an explicit formula for orthonormal Bernstein polynomials of $\mathrm{m}^{\text {th }}$ degree operational matrix of derivative. Suppose that $D$ is an $(m+1) \times(m+1)$ operational matrix of derivative. Then

$$
\frac{\mathrm{d}}{\mathrm{d} x} \mathrm{OB}(\mathrm{x}) \simeq \mathrm{D} \cdot \mathrm{OB}(\mathrm{x}), \text { where } 0 \leqslant x \leqslant 1 .
$$

From (2.4), we have $\mathrm{OB}(\mathrm{x})=\mathrm{MT}_{\mathrm{m}}(\mathrm{x})$, and thus

$$
\frac{\mathrm{d}}{\mathrm{d} x} \mathrm{OB}(\mathrm{x})=M\left[\begin{array}{ccccc}
0 & 0 & 0 & \cdots & 0 \\
1 & 0 & 0 & \cdots & 0 \\
0 & 2 & 0 & \cdots & 0 \\
\vdots & \vdots & \vdots & \ddots & \vdots \\
0 & 0 & 0 & \cdots & \mathrm{m}
\end{array}\right]\left[\begin{array}{c}
0 \\
1 \\
x \\
\vdots \\
x^{\mathrm{m}-1}
\end{array}\right]=M \wedge^{\prime} X^{\prime}
$$

where $\Lambda^{\prime}$ is $(m+1) \times m$ matrix

$$
\Lambda^{\prime}=\left[\begin{array}{ccccc}
0 & 0 & 0 & \cdots & 0 \\
1 & 0 & 0 & \cdots & 0 \\
0 & 2 & 0 & \cdots & 0 \\
\vdots & \vdots & \vdots & \ddots & \vdots \\
0 & 0 & 0 & \cdots & m
\end{array}\right]
$$

and $X^{\prime}=\left[\begin{array}{lllll}1 & x & x^{2} & \cdots & x^{m-1}\end{array}\right]^{\top}$

Now, we expand vector $X^{\prime}$ in terms of $\left\{\mathrm{OB}_{j, m}\right\}_{j=0}^{m}$. Using (2.8), we can write $X^{\prime}=B^{\prime} O B(x)$, where

$$
B^{\prime}=\left[\begin{array}{c}
M_{1}^{-1} \\
M_{2}^{-1} \\
M_{3}^{-1} \\
\vdots \\
M_{m}^{-1}
\end{array}\right]
$$

and so

$$
\frac{\mathrm{d}}{\mathrm{d} x} \mathrm{OB}(\mathrm{x})=\mathrm{M} \wedge^{\prime} \mathrm{B}^{\prime} \cdot \mathrm{OB}(\mathrm{x})
$$

Hence, we have the operational matrix of derivative as $D=M \Lambda^{\prime} B^{\prime}$.

\subsection{OBPs operational matrix of product}

In this section, we will derive an explicit formula for orthonormal Bernstein polynomials of $\mathrm{m}^{\text {th }}$ degree operational matrix of product. Assume that $c$ is an arbitrary $(m+1) \times 1$ matrix. Then $\hat{C}$ is an $(m+1) \times$ $(m+1)$ operational matrix of product whenever

$$
\mathrm{c}^{\top} \mathrm{OB}(x) \mathrm{OB}(x)^{\top} \simeq \mathrm{OB}(x)^{\top} . \hat{\mathrm{C}} \text {. }
$$

It follows from (2.4) and $c^{\top} \mathrm{OB}(x)=\sum_{j=0}^{m} c_{j} \mathrm{OB}_{j, m}(x)$ that

$$
\begin{aligned}
c^{\top} \mathrm{OB}(x) \mathrm{OB}(x)^{\top} & =c^{\top} \mathrm{OB}(x) \mathrm{T}_{m}(x)^{\top} M^{\top} \\
& =\left[c^{\top} \mathrm{OB}(x), x\left(c^{\top} \mathrm{OB}(x)\right), x^{2}\left(c^{\top} \mathrm{OB}(x)\right), \ldots, x^{m}\left(c^{\top} \mathrm{OB}(x)\right)\right] M^{\top} \\
& =\left[\sum_{k=0}^{m} c_{k} \mathrm{OB}_{k, m}(x), \sum_{k=0}^{m} c_{k} x \mathrm{OB}_{k, m}(x), \ldots, \sum_{k=0}^{m} c_{k} x^{m} \mathrm{OB}_{k, m}(x)\right] M^{\top} .
\end{aligned}
$$

Now, we approximate all functions $x^{j} \mathrm{OB}_{k, m}(x), j=0,1, \ldots, m$ in terms of $\left\{\mathrm{OB}_{j, m}\right\}_{j=0}^{m}$. Let

$$
e_{j, k}^{T}=\left[e_{0}^{j, k}, e_{1}^{j, k}, \ldots, e_{m}^{j, k}\right] .
$$


By (2.5) and (2.6), we have

$$
x^{j} \mathrm{OB}_{k, m}(x)=e_{j, k}^{\top} \mathrm{OB}(x), \quad j, k=0,1, \ldots, \mathrm{m} .
$$

Thus, we obtain

$$
\begin{aligned}
\sum_{k=0}^{m} c_{k} x^{j} \mathrm{OB}_{k, m}(x) & =\sum_{k=0}^{m} c_{k}\left(\sum_{i=0}^{m} e_{i, k}^{\top} \mathrm{OB}_{i, m}(x)\right) \\
& =\sum_{i=0}^{m} \mathrm{OB}_{i, m}(x)\left(\sum_{k=0}^{m} c_{k} e_{i, k}^{\top}\right) \\
& =\mathrm{OB}(x)^{\top}\left[e_{j, 0}, e_{j, 1}, \ldots, e_{j, m}\right] c \\
& =O B(x)^{\top} \hat{E}_{j+i},
\end{aligned}
$$

where $\hat{E}_{j+i}=\left[e_{j, 0}, e_{j, 1}, \ldots, e_{j, m}\right] c$. Defining the matrix $\hat{E}=[\hat{E}]_{(m+1) \times(m+1)}=\left[\hat{E}_{1}, \hat{E}_{2}, \ldots, \hat{E}_{m}\right]$ and substituting (2.9) into (2.10), we have

$$
\mathrm{c}^{\top} \mathrm{OB}(x) \mathrm{OB}(x)^{\top} \simeq \mathrm{OB}(x)^{\top} \hat{\mathrm{E}} M^{\top},
$$

and so

$$
\hat{C}=\hat{E} M^{\top}
$$

\section{OBPs operational matrix for solving the integro-differential parabolic equation with purely nonlo- cal conditions}

Since integral conditions are not homogeneous, it is convenient to convert the problem (1.1)-(1.3) to an equivalent problem with the homogeneous integral conditions. For this reason, we introduce a new function $u(x, t)$ representing the deviation of the function $v(x, t)$ as

$$
u(x, t)=v(x, t)-w(x, t), \quad 0<x<1, \quad 0<t \leqslant T,
$$

where

$$
w(x, t)=6(2 q(t)-r(t)) x-2(3 q(t)-2 r(t)) .
$$

The problem (1.1)-(1.3) with non-homogeneous integral conditions (1.3) can be equivalently reduced to the problem of finding a function $u$ satisfying

$$
\begin{aligned}
u_{t}(x, t)-u_{x x}(x, t) & =\int_{0}^{t} a(t-\tau) u(x, \tau) d \tau, \quad 0<x<1, \quad 0<t \leqslant T, \\
u(x, 0) & =f(x), \quad 0<x<1, \\
\int_{0}^{1} u(x, t) d x & =0, \quad \int_{0}^{1} x u(x, t) d x=0, \quad 0<t \leqslant T,
\end{aligned}
$$

where

$$
f(x)=\Phi(x)-w(x, 0)
$$

3.1. Solution of the problem

It follows from

that

$$
u_{x x}(x, t)=u_{t}(x, t)-\int_{0}^{t} a(t-\tau) u(x, \tau) d \tau
$$

$$
u_{x}(x, t)=u_{x}(0, t)+\int_{0}^{x} u_{x x}(s, t) d s=\int_{0}^{x}\left[u_{t}(s, t)-\int_{0}^{t} a(t-\tau) u(s, \tau) d \tau\right] d s,
$$

and so

$$
u(x, t)=u(0, t)+\int_{0}^{x} u_{x}(s, t) d s
$$


Therefore, we get

$$
\begin{aligned}
u(x, t) & =u(0, t)+x u_{x}(0, t)+\int_{0}^{x} \int_{0}^{s}\left[u_{t}(s, t)-\int_{0}^{t} a(t-\tau) u(x, \tau) d \tau\right] d s d s \\
& =u(0, t)+x u_{x}(0, t)+z(x, t)
\end{aligned}
$$

where

$$
z(x, t)=\int_{0}^{x} \int_{0}^{s}\left[u_{t}(s, t)-\int_{0}^{t} a(t-\tau) u(x, \tau) d \tau\right] d s d s .
$$

From (3.2) and (3.3), we obtain

$$
u(0, t)+\frac{1}{2} u_{x}(0, t)+\int_{0}^{1} z(x, t) d x=0
$$

and

$$
\frac{1}{2} u(0, t)+\frac{1}{3} u_{x}(0, t)+\int_{0}^{1} x z(x, t) d x=0
$$

Hence, we conclude that

$$
u(0, t)=6 \int_{0}^{1} x z(x, t) d x-4 \int_{0}^{1} z(x, t) d x
$$

and

$$
u_{x}(0, t)=-12 \int_{0}^{1} x z(x, t) d x+6 \int_{0}^{1} z(x, t) d x .
$$

Using (3.4) and (3.5) in (3.3), we have

$$
u(x, t)=(6-12 x) \int_{0}^{1} x z(x, t) d x+(-4+6 x) \int_{0}^{1} z(x, t) d x+z(x, t)
$$

which implies that

$$
\frac{\partial}{\partial t} \mathfrak{u}(x, t)=\frac{\partial}{\partial t}\left[(6-12 x) \int_{0}^{1} x z(x, t) d x+(-4+6 x) \int_{0}^{1} z(x, t) d x+z(x, t)\right]
$$

and thus

$$
u(x, t)-u(x, 0)=\int_{0}^{t} \frac{\partial}{\partial t}\left[(6-12 x) \int_{0}^{1} x z(x, t) d x+(-4+6 x) \int_{0}^{1} z(x, t) d x+z(x, t)\right] d t
$$

Writing the latter equation in the form

$$
u(x, t)=f(x)+\int_{0}^{t} \frac{\partial}{\partial t}\left[(6-12 x) \int_{0}^{1} x z(x, t) d x+(-4+6 x) \int_{0}^{1} z(x, t) d x+z(x, t)\right] d t .
$$

Now, we approximate the functions that satisfy (3.6) using the orthonormal Bernstein polynomials given by (2.5):

$$
\begin{aligned}
& u(x, t)=O B^{\top}(x) \cdot C \cdot O B(t), \quad f(x)=O^{\top}(x) \cdot F \cdot O B(t), \quad 6-12 x=O B^{\top}(x) \cdot e_{1}, \\
& -4+6 x=\mathrm{OB}^{\top}(\mathrm{x}) \cdot \mathrm{e}_{2}, \quad \quad \mathrm{x}=e_{3}^{\mathrm{T}} \cdot \mathrm{OB}(\mathrm{x}), \quad \int_{0}^{1} \mathrm{OB}(\mathrm{x}) \mathrm{OB}^{\mathrm{T}}(\mathrm{x}) \mathrm{dx}=1,
\end{aligned}
$$


where $C$ and $F$ are $(m+1) \times(m+1)$ matrices, $e_{1}, e_{2}$, and $e_{3}$ are $(m+1) \times 1$ matrices, $C$ is the only unknown matrix, and the rest of the matrices are known. Moreover, we give the approximation of $z(x, t)$ as follows:

$$
\begin{aligned}
z(x, t) & =\int_{0}^{x} \int_{0}^{s}\left[u_{t}(s, t)-\int_{0}^{t} a(t-\tau) u(x, \tau) d \tau\right] d s d s \\
& =\int_{0}^{x} \int_{0}^{s}\left[O B^{\top}(x) \cdot C D \cdot O B(t)-O B^{\top}(x) \cdot C \cdot \int_{0}^{t} a(t-\tau) O B(\tau) d \tau\right] d s d s \\
& =\mathrm{OB}^{\top}(x) \cdot\left(P^{2}\right)^{\top} C D \cdot O B(t)-O B^{\top}(x)\left(P^{2}\right)^{\top} \cdot C \cdot\left[\begin{array}{c}
\int_{0}^{t} a(t-s) O B_{0, m}(s) d s \\
\int_{0}^{t} a(t-s) O B_{1, m}(s) d s \\
\vdots \\
\int_{0}^{t} a(t-s) O B_{m, m}(s) d s
\end{array}\right],
\end{aligned}
$$

where

$$
\int_{0}^{t} a(t-s) O B_{j, m}(s) d s=m_{j}^{\top} O B(t)
$$

and

$$
m_{j}=\int_{0}^{1}\left(\int_{0}^{t} a(t-s) O B_{j, m}(s) d s\right) O B(t) d t, \quad j=0,1, \ldots, m .
$$

Let $M=\left[m_{j}\right]_{(m+1) \times(m+1)}$ be the $(m+1) \times(m+1)$ matrix. Then

$$
\begin{aligned}
z(x, t) & =\mathrm{OB}^{\top}(x) \cdot\left(\mathrm{P}^{2}\right)^{\mathrm{T}} \mathrm{CD} \cdot \mathrm{OB}(\mathrm{t})-\mathrm{OB}^{\top}(\mathrm{x}) \cdot\left(\mathrm{P}^{2}\right)^{\mathrm{T}} \mathrm{CM} \cdot \mathrm{OB}(\mathrm{t}) \\
& =\mathrm{OB}^{\top}(\mathrm{x})\left(\mathrm{P}^{2}\right)^{\mathrm{T}} \mathrm{C}(\mathrm{D}-\mathrm{M}) \mathrm{OB}(\mathrm{t}) .
\end{aligned}
$$

Substituting (3.7) into (3.6) and using the operational matrix of integration and derivative, we arrive at

$$
u(x, t)=f(x)+\int_{0}^{t} \frac{\partial}{\partial t}\left[(6-12 x) \int_{0}^{1} x z(x, t) d x+(-4+6 x) \int_{0}^{1} z(x, t) d x+z(x, t)\right] d t
$$

then

$$
\begin{aligned}
& \mathrm{OB}^{T}(x) \cdot \mathrm{C} \cdot \mathrm{OB}(\mathrm{t})=\mathrm{OB}^{\top}(x) \cdot \mathrm{F} \cdot \mathrm{OB}(\mathrm{t})+\int_{0}^{\mathrm{t}} \frac{\partial}{\partial \mathrm{t}}\left[\mathrm{OB}^{\top}(x) e_{1}\right. \\
& \times \int_{0}^{1} e_{3}^{T} \mathrm{OB}(x) \mathrm{OB}^{\top}(x)\left(P^{2}\right)^{T} \mathrm{C}(\mathrm{D}-\mathrm{M}) \mathrm{OB}(\mathrm{t}) \mathrm{dx} \\
& +\mathrm{OB}^{\mathrm{T}}(\mathrm{x}) \mathrm{e}_{2} \int_{0}^{1} \mathrm{OB}^{\mathrm{T}}(\mathrm{x})\left(\mathrm{P}^{2}\right)^{\mathrm{T}} \mathrm{C}(\mathrm{D}-\mathrm{M}) \mathrm{OB}(\mathrm{t}) \mathrm{d} \mathrm{x} \\
& \left.+\mathrm{OB}^{\top}(x)\left(\mathrm{P}^{2}\right)^{\top} \mathrm{C}(\mathrm{D}-\mathrm{M}) \mathrm{OB}(\mathrm{t})\right] \mathrm{dt} \\
& =\mathrm{OB}^{\top}(x) \cdot \mathrm{F} \cdot \mathrm{OB}(\mathrm{t})+\int_{0}^{\mathrm{t}} \frac{\partial}{\partial \mathrm{t}}\left[\mathrm{OB}^{\top}(x) e_{1} e_{3}^{\top}\right. \\
& \times\left(\int_{0}^{1} \mathrm{OB}(x) \mathrm{OB}^{\top}(x) d x\right)\left(\mathrm{P}^{2}\right)^{\mathrm{T}} \mathrm{C}(\mathrm{D}-\mathrm{M}) \mathrm{OB}(\mathrm{t}) \\
& +\mathrm{OB}^{\mathrm{T}}(x) e_{2}\left(\int_{0}^{1} \mathrm{OB}^{\mathrm{T}}(x) \mathrm{d} x\right)\left(\mathrm{P}^{2}\right)^{\mathrm{T}} \mathrm{C}(\mathrm{D}-\mathrm{M}) \mathrm{OB}(\mathrm{t}) \\
& \left.+\mathrm{OB}^{\top}(x)\left(\mathrm{P}^{2}\right)^{\mathrm{T}} \mathrm{C}(\mathrm{D}-\mathrm{M}) \mathrm{OB}(\mathrm{t})\right] \mathrm{dt}
\end{aligned}
$$




$$
\begin{aligned}
& =\mathrm{OB}^{\top}(x) \cdot \mathrm{F} \cdot \mathrm{OB}(\mathrm{t})+\int_{0}^{\mathrm{t}} \frac{\partial}{\partial \mathrm{t}}\left[\mathrm{OB}^{\top}(x) e_{1} e_{3}^{\top}\left(P^{2}\right)^{\top} \mathrm{C}(\mathrm{D}-\mathrm{M}) \mathrm{OB}(\mathrm{t})\right. \\
& +\mathrm{OB}^{\mathrm{T}}(\mathrm{x}) \mathrm{e}_{2} \mathrm{OB}^{\mathrm{T}}(1)\left(\mathrm{P}^{3}\right)^{\mathrm{T}} \mathrm{C}(\mathrm{D}-\mathrm{M}) \mathrm{OB}(\mathrm{t}) \\
& \left.+\mathrm{OB}^{\top}(x)\left(\mathrm{P}^{2}\right)^{\mathrm{T}} \mathrm{C}(\mathrm{D}-\mathrm{M}) \mathrm{OB}(\mathrm{t})\right] \mathrm{dt} \\
& =\mathrm{OB}^{\top}(x) \cdot \mathrm{F} \cdot \mathrm{OB}(\mathrm{t})+\int_{0}^{\mathrm{t}} \frac{\partial}{\partial \mathrm{t}}\left[\mathrm { OB } ^ { \top } ( x ) \left(e_{1} e_{3}^{\top}\left(\mathrm{P}^{2}\right)^{\top} \mathrm{C}(\mathrm{D}-\mathrm{M})\right.\right. \\
& \left.\left.+e_{2} \mathrm{OB}^{\top}(1)\left(\mathrm{P}^{3}\right)^{\top} \mathrm{C}(\mathrm{D}-\mathrm{M})+\left(\mathrm{P}^{2}\right)^{\top} \mathrm{C}(\mathrm{D}-\mathrm{M})\right) \mathrm{OB}(\mathrm{t})\right] \mathrm{dt} .
\end{aligned}
$$

Using the operational matrices we have

$$
\begin{aligned}
\mathrm{OB}^{\top}(x) \cdot \mathrm{C} \cdot \mathrm{OB}(\mathrm{t}) & =\mathrm{OB}^{\top}(x) \cdot \mathrm{F} \cdot \mathrm{OB}(\mathrm{t})+\int_{0}^{t} \frac{\partial}{\partial t}\left[\mathrm{OB}^{\top}(x) \cdot Y \cdot O B(t)\right] d t \\
& =\mathrm{OB}^{\top}(x) \cdot \mathrm{F} \cdot \mathrm{OB}(\mathrm{t})+\mathrm{OB}^{\top}(x) Y \int_{0}^{t} \frac{\partial}{\partial t} \mathrm{OB}(t) d t \\
& =\mathrm{OB}^{\top}(x) \cdot \mathrm{F} \cdot \mathrm{OB}(\mathrm{t})+\mathrm{OB}^{\top}(x) \cdot \mathrm{DPY} \cdot \mathrm{OB}(\mathrm{t}) \\
& =\mathrm{OB}^{\top}(x)[\mathrm{F}+\mathrm{DPY}] \mathrm{OB}(\mathrm{t})
\end{aligned}
$$

where

$$
\mathrm{Y}=\mathrm{e}_{1} \mathrm{e}_{3}^{\mathrm{T}}\left(\mathrm{P}^{2}\right)^{\top} \mathrm{C}(\mathrm{D}-\mathrm{M})+\mathrm{e}_{2} \mathrm{OB}^{\top}(1)\left(\mathrm{P}^{3}\right)^{\mathrm{T}} \mathrm{C}(\mathrm{D}-\mathrm{M})+\left(\mathrm{P}^{2}\right)^{\top} \mathrm{C}(\mathrm{D}-\mathrm{M})
$$

and so the following set of algebraic equations is obtained

$$
\mathrm{C}=\mathrm{F}+\mathrm{DPY} \text {. }
$$

\subsection{An illustrative example}

Consider the integro-differential equation

$$
u_{t}(x, t)-u_{x x}(x, t)=\int_{0}^{t} \exp (t-\tau) u(x, \tau) d \tau, \quad 0<x<1, \quad 0<t \leqslant T
$$

subject to the initial condition

$$
u(x, 0)=\sin (x), \quad 0<x<1
$$

and the purely nonlocal (integral) conditions

$$
\int_{0}^{1} u(x, t) d x=0, \quad 0<t \leqslant T, \quad \int_{0}^{1} x u(x, t) d x=0, \quad 0<t \leqslant T .
$$

In this case the exact solution is given by

$$
u(x, t)=\exp (-t) \cdot \cos (t) \cdot \sin (x), \quad 0<x<1, \quad 0<t \leqslant T .
$$

By applying the method in Section 3, for $m=3$, we have

$$
e_{1}=\left[\begin{array}{c}
2.3812 \\
-0.67082 \\
-1.9053 \\
-1.5
\end{array}\right], \quad e_{2}=\left[\begin{array}{c}
-1.852 \\
-0.22361 \\
0.51962 \\
0.5
\end{array}\right], \quad e_{3}=\left[\begin{array}{c}
0.13229 \\
0.33541 \\
0.37528 \\
0.25
\end{array}\right],
$$




$$
\begin{aligned}
& \mathrm{P}=\left[\begin{array}{cccc}
0.21875 & 0.39617 & 0.27959 & 0.16772 \\
-2.6411 \times 10^{-2} & 0.15625 & 0.28241 & 0.12578 \\
6.8193 \times 10^{-3} & -4.0344 \times 10^{-2} & 0.09375 & 0.14073 \\
-2.3623 \times 10^{-3} & 1.3975 \times 10^{-2} & -3.2476 \times 10^{-2} & 0.03125
\end{array}\right] \\
& \mathrm{D}=\left[\begin{array}{cccc}
-3.5 & -0.59161 & 0 & 0 \\
6.5077 & -2.5 & -1.5492 & 0 \\
-4.5826 & 5.4222 & -1.5 & -4.3301 \\
2.6458 & -2.2361 & 6.0622 & 7.5
\end{array}\right] \\
& \mathrm{F}=\left[\begin{array}{cccc}
8.5445 \times 10^{-2} & 7.2214 \times 10^{-2} & 5.5937 \times 10^{-2} & 3.2295 \times 10^{-2} \\
0.20979 & 0.17731 & 0.13734 & 7.9294 \times 10^{-2} \\
0.22053 & 0.18638 & 0.14437 & 8.3353 \times 10^{-2} \\
0.13909 & 0.11756 & 9.1058 \times 10^{-2} & 5.2573 \times 10^{-2}
\end{array}\right] \text {, } \\
& M=\left[\begin{array}{cccc}
0.26433 & 0.57333 & 0.55523 & 0.37407 \\
-3.6565 \times 10^{-2} & 0.15451 & 0.34229 & 0.19266 \\
1.0311 \times 10^{-2} & -4.3573 \times 10^{-2} & 8.5504 \times 10^{-2} & 0.15559 \\
-3.7303 \times 10^{-3} & 1.5763 \times 10^{-2} & -3.0933 \times 10^{-2} & 2.7258 \times 10^{-2}
\end{array}\right] \text {, } \\
& C=\left[\begin{array}{cccc}
8.4506 \times 10^{-2} & 6.9541 \times 10^{-2} & 5.2441 \times 10^{-2} & 2.9737 \times 10^{-2} \\
0.2117 & 0.18275 & 0.14446 & 8.4504 \times 10^{-2} \\
0.22034 & 0.18583 & 0.14364 & 8.2820 \times 10^{-2} \\
0.13732 & 0.11251 & 8.4455 \times 10^{-2} & 4.7742 \times 10^{-2}
\end{array}\right]
\end{aligned}
$$

Table 1 shows the absolute errors between the approximate solutions obtained for values of $\mathrm{m}=3$, and $\mathrm{m}=5$ using the operational matrix of Bernstein polynomials and the exact solutions.

Table 1: Absolute error of $v(x, t)$.

\begin{tabular}{ccc}
\hline$v(x, t)$ & $\mathrm{m}=3$ & $\mathrm{~m}=5$ \\
\hline$(0.1,0.1)$ & $7.9930 \times 10^{-3}$ & $2.2160 \times 10^{-8}$ \\
$(0.2,0.2)$ & $3.8124 \times 10^{-2}$ & $3.1023 \times 10^{-7}$ \\
$(0.3,0.3)$ & $8.8430 \times 10^{-2}$ & $4.3415 \times 10^{-7}$ \\
$(0.4,0.4)$ & $1.5591 \times 10^{-2}$ & $4.7789 \times 10^{-7}$ \\
$(0.5,0.5)$ & $2.3634 \times 10^{-2}$ & $5.3743 \times 10^{-7}$ \\
$(0.6,0.6)$ & $3.2429 \times 10^{-2}$ & $4.6544 \times 10^{-6}$ \\
$(0.7,0.7)$ & $4.1306 \times 10^{-2}$ & $5.6646 \times 10^{-6}$ \\
$(0.8,0.8)$ & $4.9465 \times 10^{-2}$ & $3.6602 \times 10^{-6}$ \\
$(0.9,0.9)$ & $5.5956 \times 10^{-2}$ & $3.0332 \times 10^{-6}$ \\
$(1.0,1.0)$ & $5.9662 \times 10^{-2}$ & $5.2351 \times 10^{-6}$ \\
\hline
\end{tabular}

It can be seen from Table 1 that the solutions obtained by the present method is nearly identical with the exact solutions. Clearly, increasing more higher the values of $m$ leads to highly accurate results.

\section{Conclusions}

In this paper, a numerical method is presented to give the approximate solution of a parabolic integrodifferential equation with purely nonlocal conditions. The method is derived by expanding the required approximate solution as the elements of orthonormal Bernstein basis. Using the operational matrix of integration and derivative, the problem can be reduced to a set of algebraic equations. A numerical example is included to demonstrate the validity and applicability of this method, and the results reveal that the method is very effective, straightforward, simple, and it can be applied to various types of problems with nonlocal conditions. 


\section{Acknowledgment}

The authors are grateful the editors and to anonymous referees for a very thorough reading of the manuscript and for their useful suggestions to improve this paper. This research is supported by NNSF of P. R. China (Grant Nos. 61503171 and 61403061), CPSF (Grant No. 2015M582091), NSF of Shandong Province (Grant No. ZR2016JL021), DSRF of Linyi University (Grant No. LYDX2015BS001), and the AMEP of Linyi University, P. R. China.

\section{References}

[1] W. T. Ang, A Method of Solution for the One-Dimensional Heat Equation Subject to Nonlocal Conditions, Southeast Asian Bull. Math., 26 (2003), 185-191. 1

[2] A. Ashyralyev, F. S. Ozesenli Tetikoglu, FDM for elliptic equations with Bitsadze-Samarskii-Dirichlet conditions, Abstr. Appl. Anal., 2012 (2012), 22 pages. 1

[3] A. Ashyralyev, O. Yildirim, A note on the second order of accuracy stable difference schemes for the nonlocal boundary value hyperbolic problem, Abstr. Appl. Anal., 2012 (2012), 29 pages.

[4] G. Avalishvili, M. Avalishvili, D. Gordeziani, On integral nonlocal boundary value problems for some partial differential equations, Bull. Georgian Natl. Acad. Sci., 5 (2011), 31-37. 1

[5] G. Avalishvili, M. Avalishvili, D. Gordeziani, On a nonlocal problem with integral boundary conditions for a multidimensional elliptic equation, Appl. Math. Lett., 24 (2011), 566-571. 1

[6] S. A. Beilin, Existence of solutions for one-dimensional wave equations with nonlocal conditions, Electron. J. Differential Equations, 2001 (2001), 8 pages. 1

[7] M. A. Bellucci, On the explicit representation of orthonormal Bernstein polynomials, ArXiv Preprint, 2014 (2014), 13 pages. 2

[8] G. Berikelashvili, N. Khomeriki, On the convergence of difference schemes for one non-local boundary-value problem, Lith. Math. J., 52 (2012), 353-362. 1

[9] G. Berikelashvili, N. Khomeriki, On a numerical solution of one nonlocal boundary value problem with mixed DirichletNeumann conditions, Lith. Math. J., 53 (2013), 367-380.

[10] A. Borhanifar, S. Shahmorad, E. Feizi, A matrix formulated algorithm for solving parabolic equations with nonlocal boundary conditions, Numer. Algorithms, 74 (2017), 1203-1221. 1

[11] A. Bouziani, Solution forte d'un problème mixte avec une condition non locale pour une classe d'équations hyperboliques [Strong solution of a mixed problem with a nonlocal condition for a class of hyperbolic equations], Acad. Roy. Belg. Bull. Cl. Sci., 8 (1997), 53-70. 1

[12] A. Bouziani, On the quasi static flexure of thermoelastic rod, Commun. Appl. Anal., 6 (2002), 549-568. 1

[13] A. Bouziani, R. Mechri, The Rothe's method to a parabolic integrodifferential equation with a nonclassical boundary conditions, Int. J. Stoch. Anal., 2010 (2010), 16 pages. 1

[14] Y. Liu, Numerical solution of the heat equation with nonlocal boundary conditions, J. Comput. Appl. Math., 110 (1999), 115-127. 1

[15] A. Merad, A. Bouziani, C. Ozel, Inversion Laplace transform for integrodifferential parabolic equation with purely nonlocal conditions, Hacet. J. Math. Stat., 44 (2015), 1087-1097. 1

[16] A. Merad, J. Martín-Vaquero, A Galerkin method for two-dimensional hyperbolic integro-differential equation with purely integralconditions, Appl. Math. Comput., 291 (2016), 386-394. 1

[17] N. Merazga, A. Bouziani, Rothe time-discretization method for a nonlocal problem arising in thermoelasticity, J. Appl. Math. Stoch. Anal., 2005 (2005), 16 pages. 1

[18] S. Mesloub, A. Bouziani, Mixed problem with integral conditions for a certain class of hyperbolic equations, J. Appl. Math., 1 (2001), 107-116. 1

[19] H. Qin, C. Zhang, T. Li, Y. Chen, Controllability of abstract fractional differential evolution equations with nonlocal conditions, J. Math. Computer Sci., 17 (2017), 293-300. 1

[20] J. Shen, T. Tang, L.-L. Wang, Spectral Methods: Algorithms, Analysis and Applications, Springer, New York, (2011). 2.1

[21] H. Stehfest, Numerical inversion of Laplace transforms, Comm. ACM, 13 (1970), 47-49. 1

[22] E. Tohidi, A. K1lıçman, An efficient spectral approximation for solving several types of parabolic PDEs with nonlocal boundary conditions, Math. Probl. Eng., 2014 (2014), 6 pages. 1 